\section{A reply to the critique by Marks}

\section{WILLIAM E. DAWSON and MARK E. MILLER University of Notre Dame \\ Notre Dame, Indiana 46556}

The critique of our paper by Marks (1978), an unknown reviewer to us at the time of this reply, contains a helpful further discussion of the difficulty of specifying what is causing curvature in loudness and softness functions. As such, it is totally in accord with the main thrust of our paper. On the basis of the data available to us, we concluded that the effective threshold and physiological noise models were unacceptable. Examination of the additive constant model of zero-point response bias led to more promising results, although not to a strong endorsement, as a rereading of the fourth from the last paragraph of our paper will show. Rather, we call for the collection of additional data under different conditions. We did so because data accumulating in our laboratory at the time the manuscript was written seemed to be at odds with an additive constant model, however interpreted. Research goes on, but research articles provide only static pictures of what is completed at some point in time.

Despite our agreement with the reviewer (implicit in our article) that additional experimentation may give data at odds with the additive constant model, we should like to comment on his discussion. A major fault of the zero-bias interpretation, we are told, is that the equations fitted to our data predict implausible judgments for sound pressure levels higher and lower than those employed. For example, our fitted equations predict zero and negative judgments for some SPLs. Although this is true, the reviewer overlooks the possibility that zero-point bias is context-dependent. That is, if a still wider range of SPLs were used, it could still be the case that the additive constant model would fit but that now the fitted $\mathrm{C} / \mathrm{k}$ and the implicit $\mathrm{S}_{\mathrm{O}}$ would take on new values. The value of $\mathrm{C}$ for the new set of SPLs could again be of such a magnitude that for all SPLs involved the judgments are predicted to be greater than zero. Only the further experimentation that both the reviewer and we suggested will answer this question. Some of our data still being analyzed suggests that $\mathrm{C} / \mathrm{k}$ will change with the stimulus range and that the implicit $S_{0} S$ will be driven to still smaller magnitudes for loudness and still larger ones for softness. A supporter of a zero-point bias theory might then be tempted to claim that some such bias is still operating in observers' judgments.

In ending this reply to the critique, we quote, in complete agreement, the summary statement of our reviewer: "The behavior of the subjects in judging loudness and especially softness at very high and very low SPL is still not very well understood." Nor will it be until the appropriate data is collected and examined -our reviewer's critique notwithstanding.

\section{REFERENCE}

Marks, L. E. A critique of Dawson and Miller's 'Inverse attribute functions and the proposed modifcations of the power law." Perception \& Psychophysics, 1978, 24, 569-570.

(Received and accepted for publication September 28, 1978.) 